\title{
Residual Stress Measurements In Mo/CuCrZr Tiles Using Neutron Diffraction
}

\author{
K. Mergia ${ }^{1, a}$, M. Grattarola ${ }^{2, b}$, S. Messoloras ${ }^{1, c}$, C. Gualco ${ }^{2, d}$ and M. \\ Hofmann ${ }^{3, e}$ \\ ${ }^{1}$ Institute of Nuclear Technology and Radiation Protection, National Centre for Scientific Research \\ "Demokritos", 15310 Aghia Paraskevi, Athens, Greece \\ ${ }^{2}$ ANSALDO Ricerche s.p.a., Corso Perrone 118, 16152 Genova, Italy \\ ${ }^{3}$ FRM-II, TU München, Lichtenbergstr. 1, Garching, Germany \\ akmergia@ipta.demokritos.gr, 'bmarco.grattarola@ari.ansaldo.it, 'spyros@ipta.demokritos.gr, \\ dcarlo.gualco@ari.ansaldo.it, ${ }^{\mathrm{e}}$ Michael.Hofmann@frm2.tum.de
}

Keywords: Residual stress; Brazing; Neutron diffraction; Finite Element Simulation.

\begin{abstract}
In plasma facing components (PFC) for nuclear fusion reactors tungsten or carbon based tiles need to be cooled through a heat sink. The joint between the PFC and the heat sink can be realized using a brazing process through the employment of compliant layer of either a low yield material, like copper, or a high yield material, like molybdenum. Experimental verification of the induced stresses during the brazing process is of vital importance. Strains and residual stresses have been measured in $\mathrm{Mo} / \mathrm{CuCrZr}$ brazed tiles using neutron diffraction. The strains and stresses were measured in Mo tile along the weld direction and at different distances from it. The experimental results are compared with Finite Element Simulations.
\end{abstract}

\section{Introduction}

The development of joining techniques for the fabrication of components for electronic, nuclear fusion and space applications is an important technological issue. In fusion related applications plasma facing compounds (PFC) consist of two parts: (i) the direct plasma facing material made of carbon or tungsten, and (ii) the heat sink consisting of a copper-rich $\mathrm{CuCrZr}$ alloy [1-2]. The protection material can be joined to the heat sink by means of a high temperature brazing process (up to $1040^{\circ} \mathrm{C}$ ) using silver-free alloys.

The main problem of bonding $\mathrm{W}$ or $\mathrm{C}$-material to $\mathrm{CuCrZr}$ is the large difference in their coefficient of thermal expansion (CTE). This difference creates very large residual stresses at the interface and may result in damage of the joint simply from cooling it down from the joining temperature. A low yield strength material compliant layer, like pure copper, can be introduced between the protective material and $\mathrm{CuCrZr}$ in order to lower the residual stresses by plastic deformation. Another solution is to introduce a high yield strength material interlayer with CTE matching that of the protective material, like molybdenum, in order to transfer the residual stresses from the carbon/metal interface to the strongest metal/metal one.

Neutron diffraction is a unique tool and a well-established non-destructive method for the indepth determination of residual stresses in materials, since neutrons can penetrate deep in matter [34]. The method relies on the precise measurement of the Bragg peaks at different positions within the sample so a three dimensional mapping of the residual stresses can be constructed.

In the present work neutron diffraction measurements have been employed in order to measure the strains and residual stresses in $\mathrm{Mo} / \mathrm{CuCrZr}$ brazed tiles, in the side of the Mo tile, along the weld direction and at various distances from it. Two geometries have been utilized in order to measure the normal and longitudinal stresses. The experimental results are compared with Finite Element Simulations (FES) and their discrepancies are discussed. 


\section{Experimental}

Mo tiles were brazed to $\mathrm{CuCrZr}$ ones using an active silver free filler at $1040^{\circ} \mathrm{C}$. The Mo tiles have dimensions $30 \times 30 \times 6 \mathrm{~mm}^{3}$ and the heat sink tiles $30 \times 30 \times 15 \mathrm{~mm}^{3}$. In the actual PFC the Mo tiles are expected to be thinner but, in order to be able to evaluate the strains to be transmitted to the PFC, thicker tiles were specially used for these experiments.

The neutron diffraction experiments were carried out at STRESS-SPEC instrument at FRM-II Research Reactor in Munich, Germany. The wavelength used was $1.67 \AA$ from $\operatorname{Si}(400)$ bent monochromator. The sample was placed on a X,Y,Z translation table which was attached on the top of a rotation table. The Mo (211) Bragg peak was monitored on a two dimensional position sensitive ${ }^{3} \mathrm{He}$ detector using two geometries as shown in Figs. 1a and b. The geometries depicted in Figs.1a and $\mathrm{b}$ correspond to the measurement of normal and longitudinal strain, respectively. The Mo (211) Bragg peak was followed along the weld at approximately 27 positions (Y direction) and at various distances from the weld (X direction), starting from the free Mo surface $(X=0)$ and up to a distance of $1.4 \mathrm{~mm}$ away from the weld $(X=-4.6 \mathrm{~mm})$. The gauge volume used was $1 \times 10 \times 1 \mathrm{~mm}^{3}$ with a cross-section of $1 \times 1 \mathrm{~mm}^{2}$ in the XY plane as it is shown in Figs 1a and $\mathrm{b}$. This gauge volume was positioned halfway through the Mo tile in $\mathrm{Z}$ direction. The CuCrZr Bragg peaks couldn't be monitored due to the very large grain size in $\mathrm{CuCrZr}$.

The Bragg peak position (scattering angle $2 \theta$ ) was determined by fitting the experimental profile (counts versus angle) to a Gaussian function. The $d$ - spacing of the atomic planes is calculated according to Bragg's law

$$
\lambda=2 d \sin \theta
$$

The strain, $\varepsilon$, at any measurement point was calculated using the equation

$$
\varepsilon=\frac{d^{211}-d_{0}^{211}}{d_{0}^{211}}
$$

where $d^{211}$ and $d_{0}^{211}$ are the interplanar distances between (211) planes of the stressed material and that of the free-stress material, respectively. For the determination of $d_{0}^{211}$ a free Mo tile was used. The residual stress was calculated from the strain using the generalized Hooke's laws given by

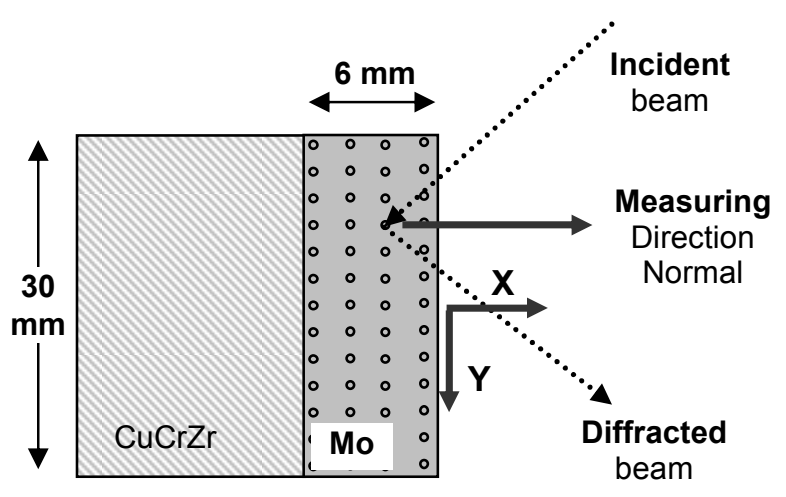

(a)

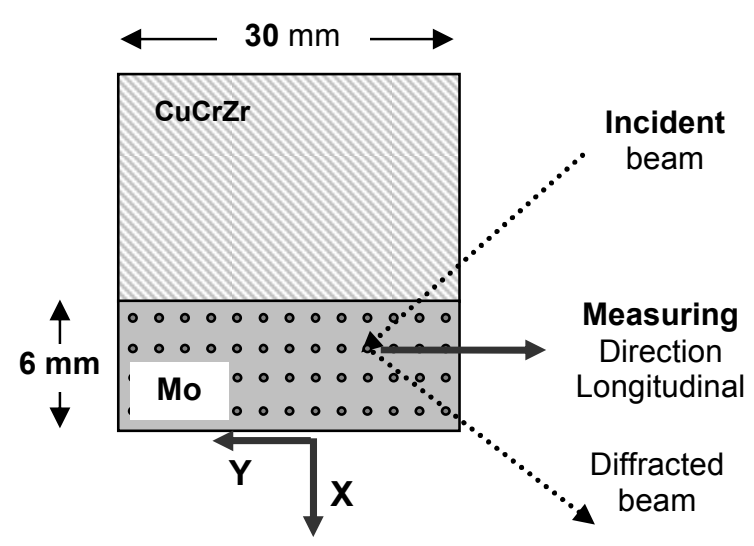

(b)

Fig. 1. The two different sample orientations used for the determination of the normal (a) and longitudinal (b) strains at different positions (indicated by the dots) within the Mo tile. 


$$
\begin{aligned}
& \sigma_{N}=\frac{E}{1+v}\left[\varepsilon_{N}+\frac{v}{1-2 v}\left(\varepsilon_{N}+\varepsilon_{L}+\varepsilon_{T}\right)\right] \\
& \sigma_{L}=\frac{E}{1+v}\left[\varepsilon_{L}+\frac{v}{1-2 v}\left(\varepsilon_{L}+\varepsilon_{N}+\varepsilon_{T}\right)\right]
\end{aligned}
$$

where $\sigma_{N}, \sigma_{L}$ the normal and longitudinal stress, respectively, and $\varepsilon_{N}, \varepsilon_{L}$ and $\varepsilon_{T}$ the normal, longitudinal and transverse strains, respectively. The transverse strain is assumed to be equal to the longitudinal one. $E$ and $v$ are the Young's modulus and the Poisson's ratio, respectively.

\section{Finite Element Simulations}

The finite element simulations (FES) were performed by means of ANSYS v.11 code. The simulation consists of a a heating phase up to $1040^{\circ} \mathrm{C}$, followed by cooling down to room temperature. During the heating phase $\mathrm{CuCrZr}$ and $\mathrm{Mo}$ are free to expand while in the successive cooling the nodes at the interface are locked to simulate brazing. Stresses generated during cooling down have been calculated according to the isotropic hardening model, using multilinear elasticplastic properties of materials. No effect of creep relaxation has been included in order to reduce numerical difficulties in this comparative analysis, although this is expected to be an important effect.

It should be noted that, due to the poor knowledge of the stress-strain curve at very high temperature $\left(>500{ }^{\circ} \mathrm{C}\right)$, the results cannot be fully representative of the real stress intensity but can only provide useful information about their distribution and about the capability of different design solutions to reduce manufacturing stresses.

\section{Results and Discussion}

The strains, in $\mu \mathrm{m} / \mathrm{m}$, and the corresponding stresses, in $\mathrm{MPa}$, both normal and longitudinal, versus the $\mathrm{Y}$ position, from the $\mathrm{Mo} / \mathrm{CuCrZr}$ brazed tile are depicted in Figs.2a-f and for various distances, X, from the Mo free surface (see Fig. 1). It is observed that both normal and longitudinal strains and stresses oscillate and they are not symmetric around $Y=0$. This asymmetry becomes enhanced as the weld is approached. The normal strains are tensile away from the weld and as we approach it they oscillate between tension and compression. A similar oscillatory behavior between tension and compression is also observed for longitudinal strains which becomes more intense close to the weld. The asymmetries around the middle of the sample can be explained by joining defects or material non-homogeneity. The maximum values for stresses away from the weld are about 180 $\mathrm{MPa}$ whereas as we approach the weld the maximum stress values increase and become $400 \mathrm{MPa}$ at the edges of the weld. There are positions along the weld where both normal and longitudinal stresses are almost zero.

In Fig. 2 are presented the results from the Finite Element Analysis concerning elastic strain (Fig. 2a) and stress (Fig. 2b) in the normal and longitudinal direction at distance $X=-4.2 \mathrm{~mm}$ from the Mo free surface (see Fig. 1). An average has been performed for the gauge volume used in the neutron diffraction experiments in order to enable a proper comparison with the experimental results. It is observed that the calculated stresses in the normal direction are tensile in the middle region and become compressive towards the edges. Their values are comparative with the experimental ones although the distribution along the weld is different. The stresses in the longitudinal direction are compressive for all $\mathrm{Y}$ positions, decreasing from the middle of the weld towards the edges. In addition, their values are much higher than the experimental ones. This discrepancy is probably due to the microstructure of the joining materials and further investigation will be carried out on the cross-section of the brazed tiles using scanning electron microscopy. Also, material properties at 
very high temperature $\left(>500^{\circ} \mathrm{C}\right)$ should be measured and in particular stress-strain curve, creep relaxation properties and thermal expansion coefficient.
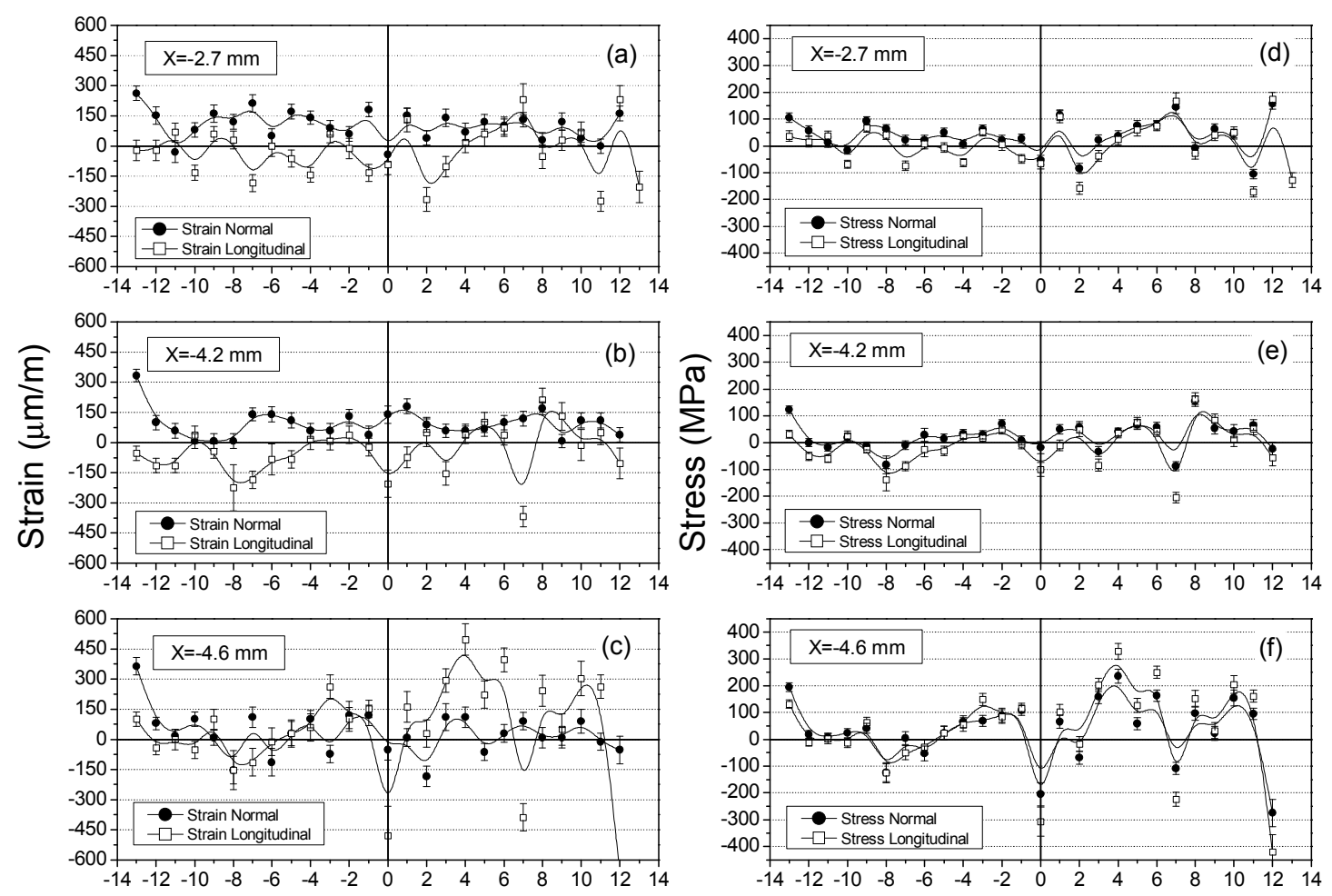

Y position

Fig. 2. Experimental normal and longitudinal strains ((a)-(c)) and stresses ((d)-(f)) for the Mo tile brazed with $\mathrm{CuCrZr}$ as a function of $\mathrm{Y}$ position along the weld and for different distances, $\mathrm{X}$, from the Mo free surface. Solid lines are guide to the eye.

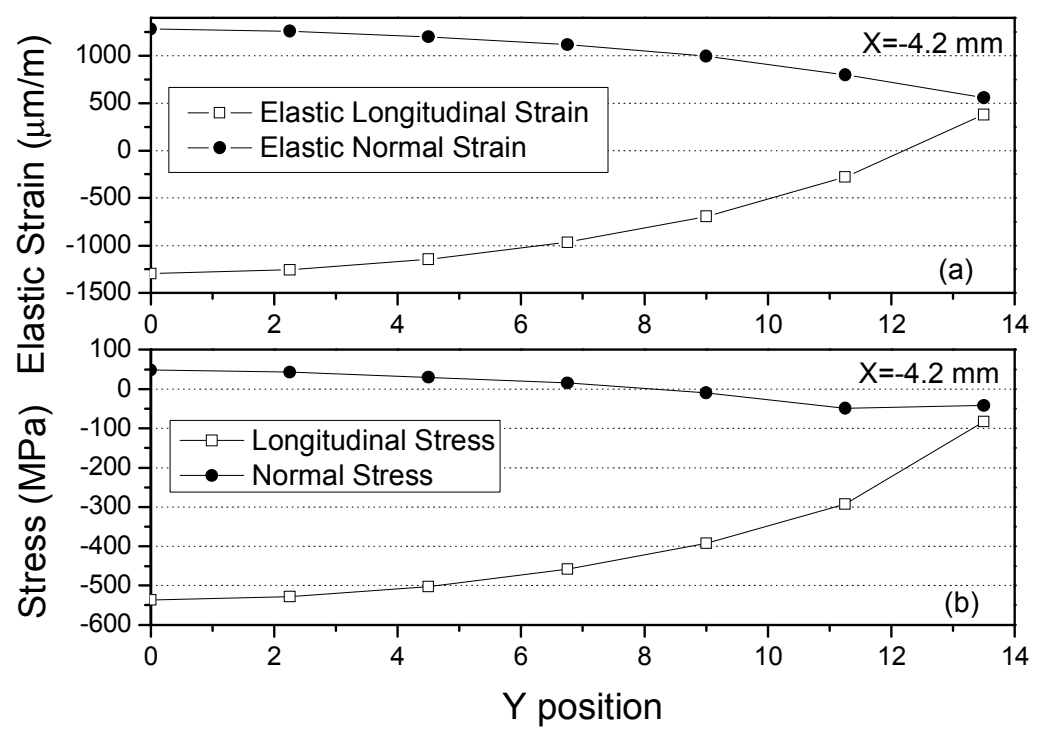

Fig. 3. The longitudinal and normal elastic strain (a) and stress (b) versus the $\mathrm{Y}$ position at distance $\mathrm{X}=$ $4.2 \mathrm{~mm}$ from the Mo free surface, as calculated by Finite Element Analysis averaged to the experimental gauge volume. Solid lines are guide to the eye. 


\section{Summary}

This study has shown that the residual stress distribution in the weld region of brazed Mo and $\mathrm{CuCrZr}$ tiles is quite complex. Although the experimental distribution of stresses along the weld differs from the calculated one, the average experimental values in the normal direction compare well with the calculated ones. On the contrary, the experimental longitudinal stresses are found to be much lower than the calculated ones. These discrepancies can be attributed to poor knowledge of the material properties at high temperatures and possibly to creep relaxation effects.

\section{References}

[1] G. Kalinin, W. Gauster, R. Matera, A.A.F. Tavassoli, A. Rowcliffe, S. Fabrietsev, H. Kawamura: J. Nucl. Mater. Vol. 233-237 (1996), p. 9

[2] A.A.F. Tavassoli: J. Nucl. Mater. Vol. 258-263 (1998), p. 85

[3] M.T. Hutchings and A. Krawitz (eds.) in: Measurement of residual and Applied Stress using Neutron Diffraction, NATO ASI Series E, Vol. 216 (1992)

[4] L. Pintschovius, in: Analysis of Residual Stress by Diffraction using Neutrons and Synchrotron Radiation, edited by M.E. Fitzpatrick and A. Lodini, Taylor \& Francis, London (2003) 